\title{
Reduced regional cerebral blood flow in Huntington's disease studied by SPECT
}

Steen G Hasselbalch, Gunilla Øberg, Sven A Sørensen, Allan R Andersen, Gunhild Waldemar, Jes F Schmidt, Kirsten Fenger, Olaf B Paulson

\begin{abstract}
Regional cerebral blood flow (rCBF) was studied in 18 patients with Huntington's disease (HD) and 19 age- and sex-matched controls with high resolution single photon emission computerised tomography (SPECT), using Tc-99m-HMPAO. Significant reductions in tracer uptake were found in the caudate and lentiform nuclei (20 and $8 \%$ ) and in the cerebral cortex, especially in the frontal and parietal areas (11-13\%). No significant reductions were found in the thalamus, mesial temporal cortex, and occipital cortex. Fourteen patients had neuropsychological testing. Relationship between rCBF and cognitive function was tested by regression analysis. A linear relationship was found between test scores of Wisconsin Card Sorting Test, Picture Arrangement Test and blood flow in the caudate nucleus. Other tests of cognitive function (Block Design Test, Face and Word Recognition Test, Street Fragmented Pictures Test, and Similarities Test) correlated better with flow in the cortical regions believed to be involved in solving those particular tests. These findings indicate, that blood flow is reduced in both cortical and subcortical structures in symptomatic HD, and that both reductions in cortical and subcortical blood flow may be related to cognitive function in $\mathrm{HD}$.
\end{abstract}

$(\mathcal{F}$ Neurol Neurosurg Psychiatry 1992;55:1018-1023)

Rigshospitalet, University State Hospital, Copenhagen Department of Neurology

$S$ G Hasselbalch G Øberg

A R Andersen

$G$ Waldemar

O B Paulson

Department of

Anaesthetics

J F Schmidt

Institute of Medical

Genetics, University of Copenhagen,

Copenhagen,

Denmark

S A Sørensen

$\mathrm{K}$ Fenger

Correspondence to:

Dr Hasselbalch, Departmen of Neurology N2081, Rigshospitalet, University State Hospital, Blegdamsve 9, DK 2100 Ø, Denmark

Received 19 March 1991 and in final revised form 9 December 1991.

Accepted 16 January 1992 symptoms, ${ }^{9}$ and cognitive function. ${ }^{10}$ These PET studies have all shown normal cortical glucose metabolism. Cortical atrophy has been found in HD by both CT and MRI. ${ }^{411}$ Studies of cortical regional cerebral blood flow have shown conflicting results of both normal blood flow $^{12}$ and reduced blood flow which correlated to the degree of dementia. ${ }^{13}$ Thus anatomical and functional mapping of the brain consistently show abnormalities in the basal ganglia, but the question of cortical involvement and its implication on cognitive functions in $\mathrm{HD}$ is not clear.

The aim of this study was twofold: 1) to find abnormal uptake patterns in subcortical or cortical structures by SPECT using Tc$99 \mathrm{~m}$-HMPAO as tracer; 2) to correlate these findings to performance in neuropsychological tests.

\section{Patients and methods}

Eighteen patients, 8 males and 10 females, were studied with SPECT; neuropsychological assessment was possible in 14 subjects (table 1). All patients were admitted from the Chorea Huntington Register at the Institute of Medical Genetics, University of Copenhagen and diagnosed on the basis of a positive family history of HD (at least one first degree relative) and typical symptoms. Median age was 46 (range 28-63 year). Patients were selected to ensure a broad representation of disease progression from recently diagnosed patients to severely affected patients. The median duration of HD was 2 years (range 1-9 years), measured from the time of diagnosis, but symptoms might have been present years before the diagnosis.

At the time of the study, twelve patients received no medicine, five patients received neuroleptics and one patient benzodiazepines. The medical therapy was not withdrawn upon entering the trial, but medication was withheld on the examination day.

The study was approved by the local ethical committee of Copenhagen and patients were included after informed consent.

The control group consisted of 19 subjects (8 males, 11 females, mean age 49 (range 23-71 years). The control group were admitted according to the protocol for a larger control study. ${ }^{14}$ Healthy subjects with no neurological deficits and a normal CT scan were included. They had no significant family history of dementia and Mini Mental State scores were all equal to or above 27.

All patients were outpatients, examined during the course of one day. After neuropsycho- 
Table 1 Patients

\begin{tabular}{lllllll}
\hline $\begin{array}{l}\text { Case } \\
\text { number }\end{array}$ & $\begin{array}{l}\text { Age } \\
\text { (years) }\end{array}$ & Sex & $\begin{array}{l}\text { Duration of } \\
\text { HD (years) }\end{array}$ & $\begin{array}{l}\text { Funct. } \\
\text { score }\end{array}$ & $\begin{array}{l}\text { Cortical } \\
\text { atrophy }\end{array}$ & $\begin{array}{l}\text { Cognitive } \\
\text { dysfunction }\end{array}$ \\
\hline 1 & 58 & M & 9 & 3 & - & ++ \\
2 & 43 & F & 3 & 5 & yes & $!$ \\
3 & 47 & F & 1 & 2 & no & + \\
4 & 55 & F & 3 & 5 & yes & $!$ \\
5 & 40 & M & 4 & 4 & yes & +++ \\
6 & 63 & M & 9 & 4 & yes & $!$ \\
7 & 47 & F & 5 & 3 & yes & +++ \\
8 & 46 & F & 1 & 2 & no & ++ \\
9 & 47 & M & 2 & 2 & no & ++ \\
10 & 57 & M & 1 & 3 & yes & + \\
11 & 53 & M & 1 & 4 & yes & $!$ \\
12 & 49 & F & 1 & 2 & no & ++ \\
13 & 41 & M & 5 & 2 & - & +++ \\
14 & 36 & F & 1 & 2 & no & +++ \\
15 & 40 & M & 1 & 1 & no & + \\
16 & 28 & F & 4 & 3 & no & ++ \\
17 & 45 & F & 2 & 2 & yes & ++ \\
18 & 37 & F & 1 & 2 & - & ++ \\
\hline
\end{tabular}

Median $46(41-51)$, values $3(1-4)=$ duration, values $3(2-3 \cdot 5)=$ funct score. Values are median values ( $95 \%$ confidence interval)

$\star$ = summed neuropsychological test scores: + mild dementia: $0-10,++$ moderate dementia: $11-20$, and +++ severe dementia: $21-\ldots !=$ could not complete the test due to advanced dementia. count values between patients and controls:

Internal ratio:

$\frac{\text { Mean countrate in region of interest }}{\text { Mean countrate in cerebellar hemisphere }} \times 100 \%$

A standard atlas method developed in our laboratory was used for placement of regions of interest (ROI). ${ }^{14}$ From a collection of consecutive sets of standard regions based on a large control material, a set was chosen for each slice. If necessary, a region of interest was redrawn to fit the contours of the brain, but the interregional relations were not altered. In all cases, the caudate nucleus region was redrawn in accordance with its size and location as judged from the CT scan. All paired CT and SPECT images were evaluated "blindly". When a ROI comprised subregions from more than one brain slice, the mean pixel value was estimated as a volume weighted mean of the values obtained from the subregions. Due to the small size of the putamen and globus pallidus, it was necessary to combine these regions into one, the lentiform nucleus. Lentiform, caudate, and thalamic flow values were obtained from the $O M+5 \mathrm{~cm}$ slice, mesial temporal area (including hippocampus) from the $\mathrm{OM}+3.6 \mathrm{~cm}$ slice.

\section{Neuropsychological test}

Included in a wider test battery were seven well known tests, chosen as probes of five aspects of congnitive functioning:

1) Reasoning/problem-solving (Wisconsin Card Sorting Test, Picture Arrangement Test).

2) Learning/recent memory (Face and Word Recognition Test).

3) Language/concept formation (WAIS Similarities Test).

4) Spatio/constructive ability (Block Design Test, 12 Four-blocks Tests).

5) Visual perception (Street Fragmented Pictures Test).

A total of 120 patients with orthopaedic and peripheral diseases, but no known cerebral dysfunction ${ }^{1920}$ served as the reference group for evaluation of individual test results (see statistics).

The degree of dementia was evaluated by the total test score, which placed the patient in one of three groups (slight, moderate, and severe dementia). Four patients who could not complete the neuropsychological test programme were evaluated by bedside cognitive tests, which in all cases confirmed the finding of severe dementia.

\section{Statistics}

Correlations between disease parameters and SPECT findings were either measured by the Mann-Whitney U-test (CT atrophy present or not present) or by Spearman Rank correlation. Regions of interest ratios were tested under the null hypothesis using the Mann-Whitney U-test and the Bonferroni correction for multiple comparisons. After correction, a probability of less than 0.05 was considered significant.

The neuropsychological test scores of each 
Figure 1 SPECT images at the level of $5 \mathrm{~cm}$ above the OM plane obtained with ${ }^{99 m}$ Tc-d,1-HMPAO

The images are scaled from 0 to 132 (relative to cerebellum $=100)$. A:

Control, 51 years old normal female. Note the indentation of the caudate nuclei into the lateral ventricles. B: Case 16, 28 year old female with $H D$ and moderate cognitive impairment. Note the reduced uptake in both caudate nuclei. Cortical uptake is only slightly reduced. C: Case 6, 63 year old male with $H D$ and a disease duration of nine years, severe dementia and diffuse cortical atrophy on CT. The lateral ventricles have the typical "box shape" due to degeneration of the caudate nuclei and cortical uptake is markedly reduced.
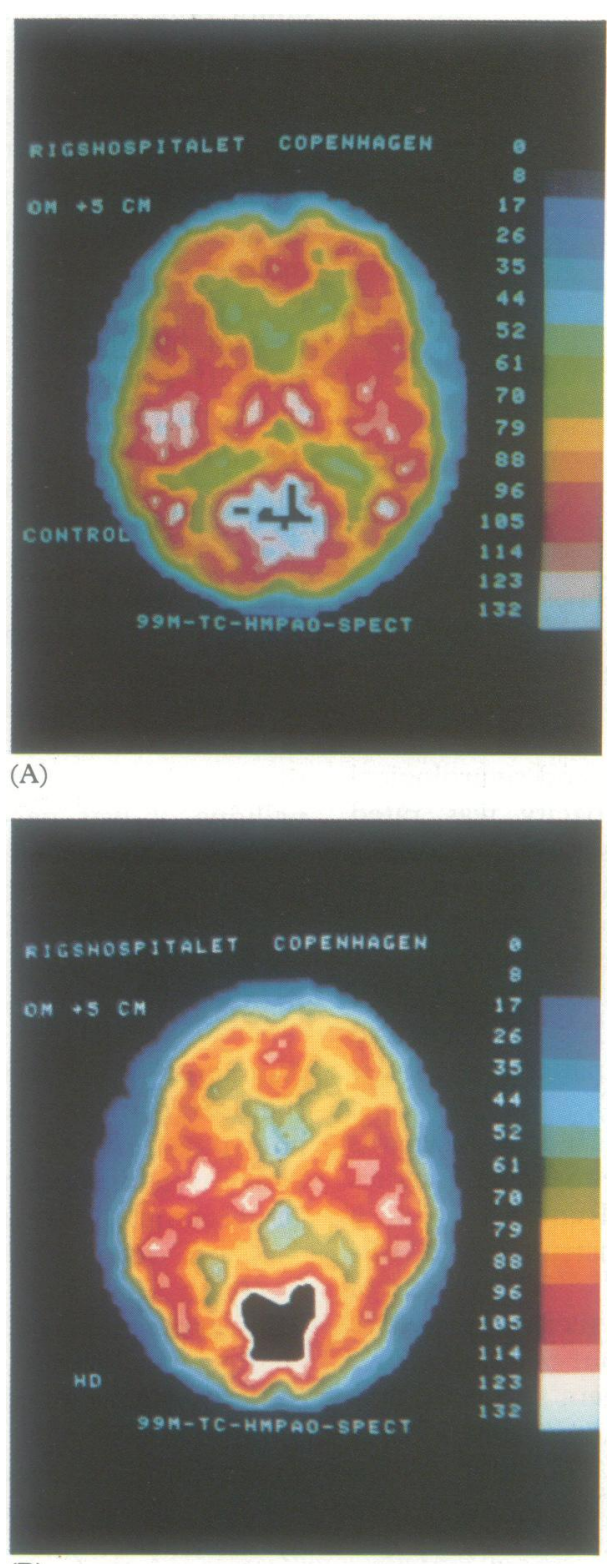

(B)

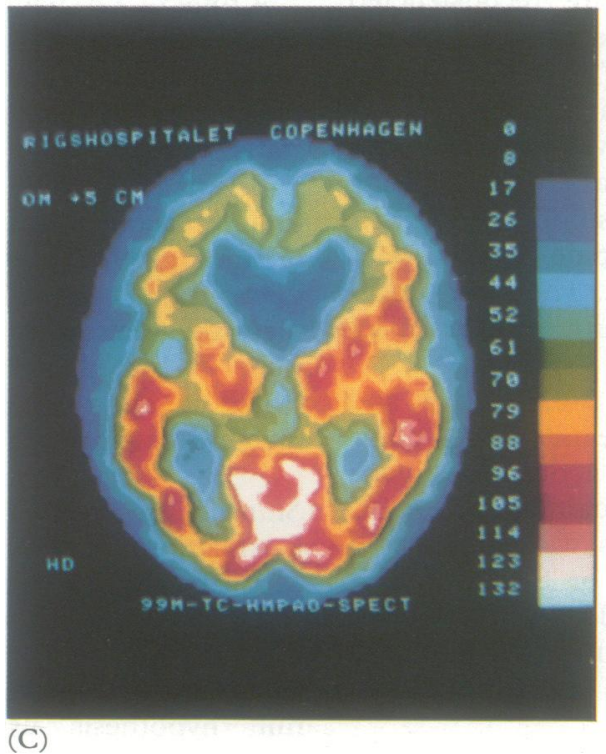

patient were compared with the control group as follows: deviations from expected test performance, based on regression analysis of the variables of age and education for the 120 reference subjects, were calculated for each test, as well as the statistical significance of the resulting difference score. ${ }^{1819}$ Thus the higher the test score, the larger was the negative deviation from expected level of performance-indicating severity of cognitive decline.

Correlation between neuropsychological test scores and regional flow values was tested by regression analysis with flow values as predictors and test score in each test as outcome.

As our preliminary analyses, using linear regression, showed that caudate flow was the best single predictor of some test performances and cortical flow variables were the best predictors of other test performances, we had to discriminate between two possible situations: a) test performance has the generally expected association with cortical blood flow, and the preliminary finding of correlation between caudate flow and test performance simply reflects the inter-regional correlation between regional blood flows - in combination with the fact that the caudate nucleus is the key target of the HD disease process, or b) the preliminary finding is genuine in the sense that test performance degrades because of reduced subcortical rather than cortical blood flow. To this end, the cortical region(s) most likely to be involved in solving a particular test was designated and used in a two-step regression, the caudate flow value being introduced into the regression equation in the second step. Whenever the second step did not add significantly to predictive power $\left(R^{2}\right.$, as judged by the appropriate $t$ statistic), there was no reason to abandon the simple hypothesis (a) in favour of idea (b).

\section{Results}

The two subgroups of HD patients with $(n=8)$ and without $(n=7)$ cortical atrophy, showed no differences in caudate flow or mean cortical flow (Mann-Whitney U-test). Duration of $\mathrm{HD}$, and functional score were not correlated with flow in the caudate nuclei or with mean cortical flow (Spearman rank correlation).

\section{Region of interest flow values}

Reduction in Tc-99m-HMPAO uptake was found in both subcortical and cortical ROIs in the HD group compared with the controls (fig 1). As expected, significant reduction was found in the caudate nuclei $(20 \%)$, smaller, but still statistically significant, reduction was found in lentiform nuclei $(8 \%$ ) (table 2 ). The reduction found in the thalamus did not reach statistical significance, and uptake in the mesial temporal region was comparable in the two groups. In all cortical regions, except in occipital cortex, statistically significant reductions in tracer uptake were found, especially in dorsolateral frontal and parietal regions (11 and $13 \%)$. The parietal cortex showed the highest variability among the cortical flow values. The total cortical flow value comprised a volume 
Table 2 Region of Interest Ratios

\begin{tabular}{lllcl}
\hline & $n$ & $H D$ & Control & p Significance \\
\hline N Caud & 18 & $77(71-81)$ & $96(91-99)$ & $<0.0001$ \\
N Lent & 18 & $94(90-98)$ & $102(99-108)$ & $<0.001$ \\
Thalam & 18 & $95(91-98)$ & $104(97-108)$ & NS \\
Mes Tmp & 16 & $83(79-85)$ & $85(83-88)$ & NS \\
V Front & 16 & $89(86-91)$ & $96(91-99)$ & $<0.03$ \\
D Front & 17 & $84(79-87)$ & $95(91-99)$ & $<0.001$ \\
Sup Tmp & 18 & $94(92-97)$ & $101(98-106)$ & $<0.03$ \\
Pariet & 17 & $84(80-90)$ & $97(96-101)$ & $<0.002$ \\
Occip & 18 & $101(96-105)$ & $101(99-110)$ & NS \\
Tot Cort & 15 & $91(88-95)$ & $97(95-102)$ & $<0.03$
\end{tabular}

Median values ( $95 \%$ confidence interval) of flow ratios ((count rate in region of interest/coun rate in cerebellar hemisphere) $\left.{ }^{\star} 100\right)$ in $\mathrm{HD}$ and control group.

$\mathbf{N}$ Caud = Caudate Nucleus, $\mathbf{N}$ Lent $=$ Lentiform Nucleus (Globus Pallidus and Putamen), Thal = Thalamus, V Front $=$ Ventral Frontal Cortex, D Front $=$ Dorsolateral Frontal

Cortex, Ms Temp = Mesial Temporal Cortex, Sup Tmp = Superor Temporal Cortex, Paret

$=$ Parietal Cortex, Occip = Occipital Cortex, Tot Cot = All Cortical ROIs.

weighted mean value of all cortical regions examined and was reduced by $6 \%$ in the HD group.

\section{Neuropsychological test scores}

The test results are shown in fig 2. A high test score corresponds to a poor performance in the test (see statistics). The most sensitive test was the WAIS Picture Arrangement which showed a significant impairment in all patients but one. Least affected was the test of visual synthesis (Street Fragmented Pictures Test), where only 4 patients had significantly high scores. The Block Design Test showed a considerable variability. Seven patients scored within the normal range in this test, whereas several subjects had marked difficulties amounting to constructional apraxia in one patient (case 7). All but one subject had significantly high scores in at least two tests; case 15 showed clearly subnormal performance only in The Picture Arrangement Test.

Four patients could not complete the tests due to severe dementia. These four patients

\section{Neuropsychological Test Scores}

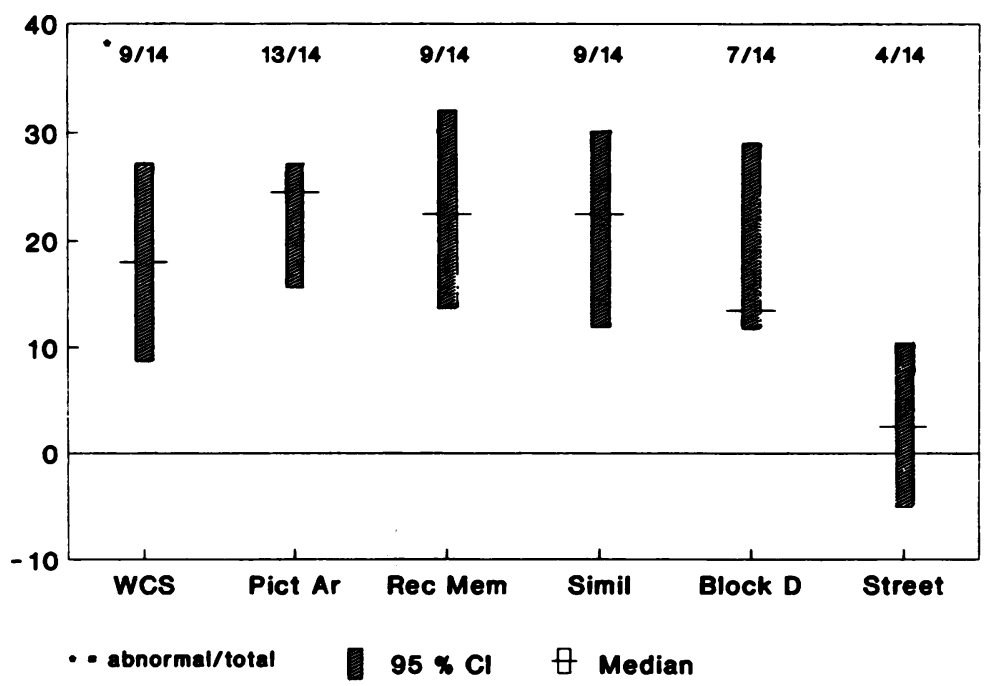

Figure 2 Neuropsychological test scores. Values are deviations from expected performance (0), a high score corresponds to a poor performance (see statistics). Lines indicate median values, bars $95 \%$ confidence intervals. The ratio on the number of patients, who had abnormal scores to the total of patients tested is shown.

WCS = Wisconsin Card Sorting Test, Pict Ar = Picture Arrangement Test, Rec Mem = Face and Word Recognition Test, Simil = WAIS Similarities Test, Block $D=$ Block Design Tests, Street = Street Fragmented Picture Test. were not included in the regression analysis of relationship between test results and regions of interest flow values since individual test results could not be obtained.

Results of the regression analysis are shown in table 3. By adding the caudate nucleus to the regression equation, the linear relationship improved significantly between the test results of Wisconsin Card Sorting Test and ROI flow values $\left(\mathbf{R}^{2}\right.$ increased from a non significant value of $27 \cdot 2 \%$ to $61 \cdot 0 \%$ ), and between Picture Arrangement Test and ROI flow values $\left(R^{2}\right.$ increased from $37 \cdot 8$ to $\left.61 \cdot 2 \%\right)$. This indicates a significant linear relationship between caudate flow values and test scores in these two tests with poor test performance corresponding to low flow values in the caudate nucleus and hence points to the caudate region as the primary source of the performance reduction (hypothesis (b) of the methods section). An insignificant ( $t$ between 1.23 and -0.32 ) increase in an already significant $R^{2}$ was found after adding caudate nucleus to the regression equation between flow values and test results of Recognition Memory Test, Similarities Test, Block Design Test, and Street Fragmented Pictures Test, indicating that there is no reason to view the caudate region as the primary source of performance reduction in the context of these four tests (note that the Similarities Test is also only slightly significantly associated with cortical flow).

\section{Discussion}

The main findings in this study are reductions in regional cerebral blood flow in both subcortical and cortical structures in symptomatic HD patients.

The lack of correlation between disease parameters such as duration of HD, functional score, and cortical atrophy with SPECT findings is probably due to the small numbers being studied, but might also reflect the variable progression of the disease.

Since the SPECT-HMPAO method measures internal ratios, a suitable reference region is required, that is, a region that is easily defined and not affected by the disease studied. In diseases affecting both cortical and subcortical structures, any kind of cortical reference region diminishes the difference between HD patients and controls, since abnormalities are found in both the region of interest and the reference region. Although the occipital cortex seems less affected in HD than other cortical areas, this region is difficult to define exactly on a SPECT image. The cerebellum, however, is easily depicted. A loss of Purkinje cells and neural depletion have been found in the cerebellum in $\mathrm{HD},{ }^{20}$ but there seems to be no substantial loss of cerebellar neurons. ${ }^{21}$ In 14 of the HD patients we measured rCBF with the $\mathrm{Xe}^{133}$ inhalation technique. Since this technique yields image resolution of only approximately $2 \mathrm{~cm}$ we considered it unsuitable for a region of interest analysis, and data are therefore not shown. The absolute flow, mean (SD), in the cerebellar hemispheres was, however, measured and was comparable in the 
Table 3 Relationship between neuropsychological test results and regional cerebral blood flow

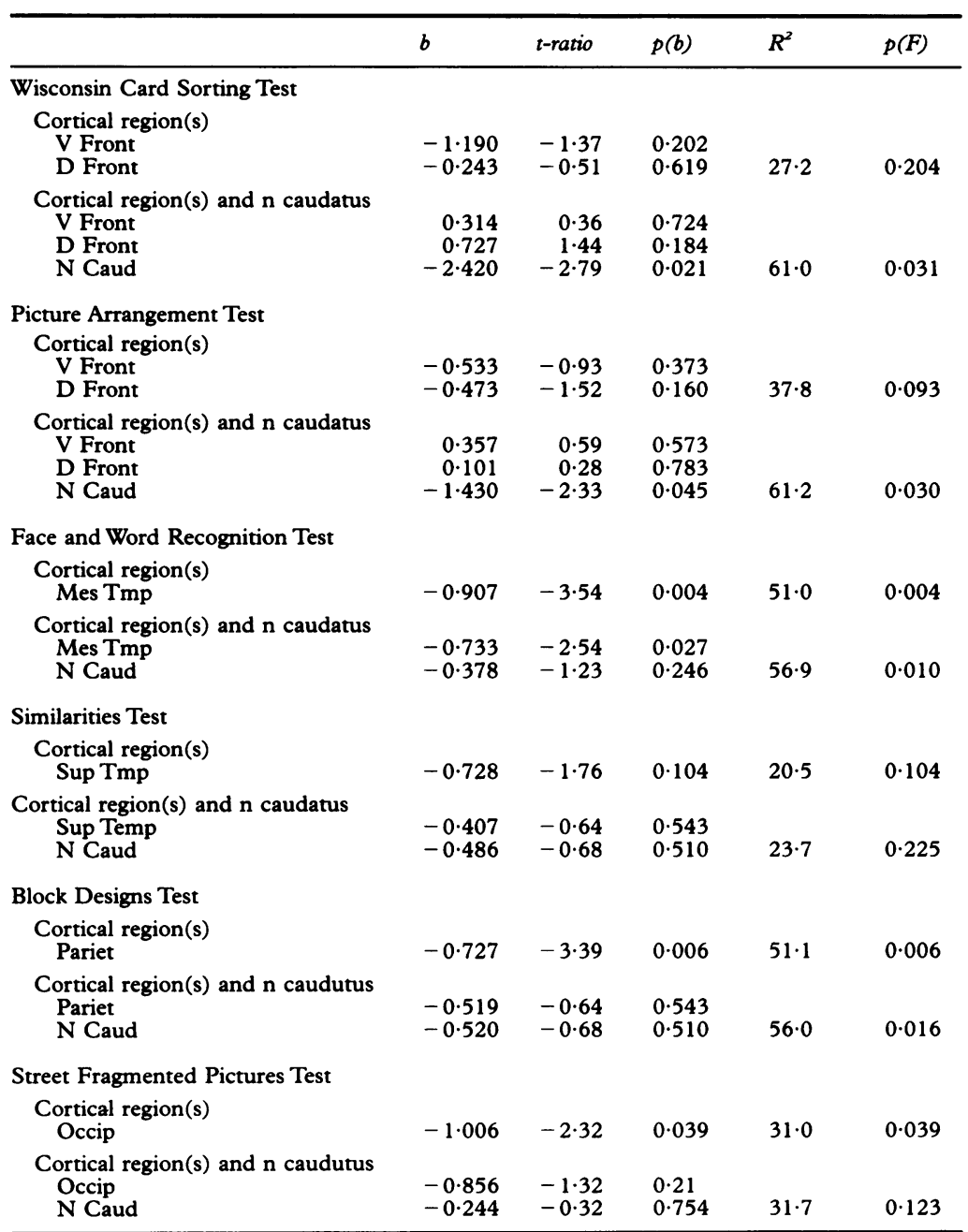

The cortical region(s) which were believed to be involved in solving a particular test were analysed by multiple (single) regression analysis with neuropsychological test results as outcome and $r C B F$ as predictors. Then $r B C F$ in caudate nucleus was included as an additional predictor (see statistics). The four patients who could not complete the neuropsychological tests were excluded from this analysis. Shown are $b=$ regression coefficient (negative value indicate correlation between poor test performance and low flow values), $t$-ratio $=b /$ standard error of $b, p(b)=$ probability of $b=0(t$-test $), R^{2}=\%$ of variability explained by the regression measured by analysis of variance, and

$p(F)=$ probability of no linear relationship for the multiple (single) regression equation $p(F)=$ probability of no linear relationship for the multiple (single) regression equation
$(F-$ test $)$. In cases of single regression analysis $p(b)=p(F)$.V Front $=$ Ventral Frontal Cortex, D Front = Dorsolateral Frontal Cortex, $N$ Caud = Nucleus Caudatus, Mes

Temp = Mesial Temporal Cortex, Sup Tmp = Superior Temporal Cortex,

Pariet $=$ Parietal Cortex, occip $=$ occipital cortex. and with a similar method, Tanahashi ${ }^{13}$ found significant flow reductions in mean hemispheric flow of aproximately $15 \%$, most pronounced in the frontotemporal and parietal regions. We confirm these findings by Tanahashi, in terms of relative activity. Measuring glucose metabolism with PET in HD patients, Kuhl et al, Hayden et al, and Young et al ${ }^{7-9}$ found a much more profound reduction in caudate metabolism of approximately 50 to $60 \%$, compared with $20 \%$ flow reduction found in this study. We used a standard atlas ROI approach, which might increase flow values, because regions can be redrawn to avoid incorporating large sulci or ventricles in the ROI. In contrast to ROIs of fixed shape (that is, circles or boxes) our approach does, to some extent, eliminate the effect of partial volume, which is particularly important to take into account when measuring blood flow in neurodegenerative diseases. Apart from differences in methods, differences in patient material could explain the discrepancy between this study and the PET studies, since eight of our patients had a disease duration of less than one year. The SPECT method, as mentioned earlier, measures internal ratios, and the problem of reference region might also have had an influence on the results. The activity distribution images can be corrected for early backdiffusion of the tracer by the method proposed by Lassen et al. ${ }^{22}$ In our study, this approach would further decrease the flow in caudate nuclei by approximately $8 \%$ to a total decrease of $28 \%$.

Relationship between neuropsychological test scores and flow values

Flow in the caudate nuclei seemed to be related to the test scores of the Wisconsin Card Sorting Test and the Picture Arrangement Test. Although these two tests require frontal-type abstract reasoning, and flow was clearly subnormal in frontal cortical regions, the cortical flow values were less related to the test scores of these tests. Although rCBF is a crude measure of neuronal function, these findings might support the hypothesis that the frontal dysfunction found in HD is due to degeneration of the caudate nuclei and not directly related to some intrinsic malfunction of the frontal cortex. This hypothesis is also supported by CT studies, in which caudate atrophy correlated to functional status ${ }^{5}$ and to cognitive impairment, ${ }^{6}$ and by PET studies, in which caudate metabolism correlated to overall functional capacity and cognitive function, and putamen metabolism to motor symptoms. ${ }^{910}$ However, a counter hypothesis could be that any intrinsic malfunction of the frontal (and functionally related) cortex is better reflected by caudate than cortical flow values because the former have a closer correlation with the disease stage and/or have less error of measurement. This hypothesis is, however, not supported by our findings with the other four neuropsychological tests. There we found that test scores (possibly apart from Similarities Test) were significantly related to cortical flow values and only indirectly to caudate flow 
values. It is tempting to hypothesise that the cognitive dysfunction in HD stems from both subcortical and cortical degeneration.

To evaluate the four severely demented patients who could not complete the neuropsychological tests, we assigned a total test score of 40 (above the rest of the group) for these patients, ranked test scores and flow values, and tested the validation of the regression analysis once more. This gave essentially the same results, although the correlation between test results and rCBF became stronger. As an example, $\mathbf{R}^{2}$ for the regression analysis between Wisconsin Card Sorting Test scores and frontal cortical flow values improved from $27 \cdot 2$ to $36 \cdot 6 \%$. By including caudate flow values into this regression analysis, $\mathbf{R}^{2}$ increased from 61.0 to $72.5 \%$. This indicates that our conclusion regarding the regression analysis is not altered by including the severely demented patients.

It may be an overinterpretation of our data to claim that "frontal" functions are those most affected by lesions in the caudate nuclei, although this is the pattern we observed. Also, in three patients with severe cortical flow reduction and diffuse cortical atrophy, clear constructional apraxia, aphasia and typical "frontal" behaviour was found (cases 4,6 , and 7). In a recent study, Kuwert et $a l^{23}$ reported that both cortical and caudate glucose metabolism correlated to overall cognitive performance in severely affected HD patients, which agrees with the findings of this study. Thus whatever might be the cause and extent of this degeneration of cortex, it might eventually reach a stage where it contributes significantly to the cognitive dysfunction found in HD.

This study was supported by grants from The Lundbeck Foundation, The Danish Medical Research Council, The Wachenhausen Foundation, and The Folmer and Ingeborg Lüttichau Foundation. We thank Dr Jorgen Hilden, Institute of Medical Genetics, University of Copenhagen, for valuable discussion and Gerda Thomson for expert technical assistance.

1 Conneally PM. Huntington's disease: Genetics and epidemiology. Am f Hum Genet 1984;36:506-26.

2 Vonsattel J-P, Myers RH, Stevens TJ, et al. Neuropathological classification of Huntington's disease. $\mathcal{F} \mathrm{Neu}$ ropathol Exp Neurol 1985;44:559-77.

3 Gusella JF, Wexler NS, Conneally PM, et al. A polymorphic DNA marker genetically linked to Huntington's disease.
Nature 1983;306:234-8.

4 Starkstein SE, Folstein SE, Brandt J, et al. Brain atrophy in Huntington's disease. A CT-scan study. Neuroradiology 1989;31:156-9.

5 Stober T, Wussow W, Schrimgrigk K. Bicaudate diameterthe most specific and simple CT parameter in the diagnosis of Huntington's disease. Neuroradiology diagnosis of

6 Bamford A, Caine ED, Kido DK, et al. Clinical-pathological correlation in Huntington's disease: A neuropsychological and computed tomography study. Neurology 1989;39:796-801.

7 Kuhl DE, Phelps ME, Markham CH, et al. Cerebral metabolism and atrophy in Huntington's disease by 18-FDG and computed tomographic scan. Ann Neurol 1982;12:425-34.

8 Hayden MR, Martin WR, W, Stoessl AJ, et al. Positron emission tomography in the early diagnosis of Huntington's disease. Neurology 1986;36:888-94.

9 Young AB, Penney JB, Starosta-Rubinstein S, et al. PET scan investigations of Huntington's disease: Cerebral scan investigations of Huntington's disease: Cerebral tional decline. Ann Neurol 1986;20:296-303.

10 Berent S, Giordani B, Lethinen S, et al. Positron emission tomographic scan investigations of Huntington's disease: Cerebral metabolic correlates of cognitive function. $A n n$ Neurol 1988;23:541-6.

11 Simmons JT, Pastakia B, Chase TN, et al. Magnetic resonance imaging in Huntington's disease. AfNR 1986;7:25-8.

12 Weinberger DR, Berman KF, Iadarola M, et al. Prefrontal cortical blood flow and cognitive function in Huntington's disease. $\mathcal{f}$ Neurol Neurosurg Psychiatry 1988;51: ton's disea.

13 Tanahashi N, Meyer JS, Ishikawa Y, et al. Cerebral blood flow and cognitive testing correlate in Huntington's flow and cognitive testing correlate
disease. Arch Neurol 1985;42:1169-75.

14 Waldemar G, Hasselbalch SG, Andersen AR, et al. $\left[{ }^{99 \mathrm{~m}} \mathrm{Tc}\right.$ ] HMPAO and SPECT of the brain in normal aging. $\mathcal{J}$ Cereb Blood Flow Metabol 1991;11:508-21.

15 Shoulson I. Huntington's disease: Functional capacities in patients treated with neuroleptic and antidepressan drugs. Neurology 1981;31:1333-5.

16 Stokely EM, Sveinsdottir E, Lassen NA, Rommer P. Single photon dynamic computer assisted tomograph (DCAT) for imaging brain function in multiple sections. $\mathcal{f}$ Comput Assist Tomogr 1980;4:230-40.

17 Celsis $\mathrm{P}$, Goldman T, Henriksen L, Lassen NA. A method for calculating regional cerebral blood flow from emission computed tomography of inert gas concentrations. $f$ Computed tomography of inert gas

18 Gade A, Mortensen EL, Udesen M, et al. On the importance of control data and background variables in the evaluation of neuropsychological aspects of brain functioning. In: Neurobehavioural methods in occupational and environmental health. Environmental Health Series. Copenhagen: WHO, 1985:91-6.

19 Gade A, Mortensen L, Bruhn P. "Chronic painter's syndrome". A reanalysis of psychological test data in a group of diagnosed cases, based on comparisons with matched controls. Acta Neurol Scand 1988;77:293-306.

20 Jeste DV, Barban L, Parisi J. Reduced Purkinje cell density in Huntington's Disease. Exp Neurol 1984;85:78-86.

21 Bedal MF, Swartz KJ, Finn SF, et al. Amino acid and neuropeptide neurotransmitters in Huntington's disease neuropeptide neurotransmitters in Hun
cerebellum. Brain Res 1989;454:393-6.

22 Lassen NA, Andersen AR, Friberg L, Paulson OB. The retention of Tc99m-D, L-HMPAO in the human brain after intracarotid bolus injection: a kinetic analysis. $\mathcal{f}$ Cereb Blood Flow Metab 1988;8(suppl 1):S13-22.

23 Kuwert T, Lange HW, Langen J, Herzog H, Aulich A, Feinendegen LE. Cortical and subcortical glucose consumption measured by PET in patients with Huntington's disease. Brain 1990;113(Pt 5):1405-23. 